\title{
Midline Nasal Dermoid - A Series of Thirteen Cases and Review of Literature
}

\author{
Vedula Padmini Saha, ${ }^{1}$ Debangshu Ghosh, ${ }^{2}$ Santanu Dutta, ${ }^{3}$ Somnath Saha, ${ }^{4}$ Sumit Kumar Basu ${ }^{2}$
}

\section{Introduction:}

\section{ABSTRACT}

Congenital midline nasal masses include nasal dermoid sinus cysts, encephaloceles and gliomas. They are rare malformations, of which dermoid cyst is a relatively common congenital abnormality. A systematic review of the clinical feature and management outcome of congenital midline nasal dermoids would help in predicting the outcome of such cases.

\section{Materials and Methods}

A prospective observation was made on a series of patients with nasal dermoids with or without sinus tract for a period of three years. Altogether thirteen patients were evaluated. Age of the patients ranged from two to twenty seven years. There were seven males and six females. Intracranial extension was ruled out radiologically. Excision of the dermoid cyst with or without sinus tract was undertaken under general anesthesia and followed up for one year.

\section{Conclusion}

Surgery is the gold standard treatment for nasal dermoids and complete excision and cosmetically good repair is mandatory for good results.

Keywords:

Dermoid Cyst; Nose

$\mathrm{I}$ solated congenital anomalies of the nose are very rare despite its complicated embryological development and intimate association with development of the face and brain. ${ }^{1}$ Many fetuses die in utero due to severe structural abnormalities in this region. ${ }^{2}$ Developmental anomalies of the nose encompass a diverse group of conditions. Embryologic developmental anomalies of the nose consist of congenital midline nasal masses (CMNMs) that include nasal dermoid sinus cysts (NDSC), gliomas and encephaloceles. They are rare malformations of which dermoid cyst is a relatively common congenital abnormality. ${ }^{1,3,4,5}$ Others are

1 - Department of Plastic and Reconstructive Surgery, $R G$ Kar Medical College, Kolkata

2 - Department of ENT, Medical College, RG Kar Medical College, Kolkata

3 - Department of ENT, Chinsurah District Hospital,

Hooghly

4 - Department of ENT, NRS Medical College, Kolkata

\section{Corresponding author:}

Dr Debangshu Ghosh

email: ghoshdr.d777@ymail.com nasal clefts, proboscis lateralis, arhinia, polyrrhinia, supernumerary nostrils, nasopharyngeal teratoma, and epignathus. ${ }^{4,6}$ They may also be a part of BeckwithWiedemann syndrome and other associated anomalies. ${ }^{5,7}$ Congenital nasal deformities were classified into four categories. ${ }^{8}$

Type I, hypoplasia and atrophy, represents paucity, atrophy, or underdevelopment of skin, subcutaneous tissue, muscle, cartilage, and/or bone. Type II, hyperplasia and duplications, representing anomalies of excess tissue, ranging from duplications of parts to complete multiples, are categorized here. In the type III category, clefts where the comprehensive and widely utilized Tessier classification of craniofacial clefts is applied. Type IV deformities consist of neoplasms and vascular anomalies.

Management of congenital nasal defect requires surgery and a team work encompassing skills from different specialities like Radiology, Maxillofacial surgery, Neurosurgery, Otolaryngology, Plastic surgery etc. Because of rarity of incidence and cosmetic challenge, these patients present a great challenge to the practising Otolaryngologist. Nasal dermoids are the 
most common congenital nasal anomaly encountered in ENT clinics. ${ }^{3,4}$

In this article we share, discuss and review the cases of midline nasal dermoids and their management at a tertiary care hospital to compare their pre and postoperative results and surgical complications.

\section{Materials and Methods}

The study was conducted from June 2009 to May 2012. Patients who presented at ENT OPD with midline nasal mass causing cosmetic deformity with or without sinus or abscess and discharge thereof were taken up for the study. A total of thirteen such patients were observed whose age ranges from two to twenty seven years. Among the patients, seven were males and six females and male:female ratio being almost equal (1.2:1). Computed tomography (CT) scan was performed in all patients to evaluate the disease and to follow its extension if any. All of them were found to have soft tissue shadow in between the two nasal bones and there was definite separation of the two nasal bones at different levels due to the cyst, sinus or soft tissue mass in the scan.

All the patients and/or parents were counselled for surgery and informed consent was taken for surgery as well as for medical photography (both preoperative, intraoperative and postoperative) on special forms. All

the patients were followed up for a period of one year postoperatively for any complications or recurrence and these, if any were noted at the end of one year postoperatively. Some cases deserve special mention.

One twenty seven year old male patient came for the treatment of recurrence of the nasal dorsal mass after a surgery done elsewhere two years back (Fig.1).

Another seven year old girl presented with infected cyst like swelling over dorsum of nose, who was diagnosed clinically as infected midline nasal dermoid with abscess formation and underwent incision and drainage followed by antibiotic treatment. After control of infection she was evaluated radiologically and prepared for surgery. One two year old boy presented with a cyst and a sinus simultaneously over the dorsum of nose. Among others three patients presented with cyst (Fig.2) and the rest with sinus tract over the dorsum of nose (Fig.3).

One patient presented with recurrence and was advised to do a sinogram preoperatively which showed persistence of sinus tract and soft tissue mass over dorsum of nose without having any intracranial connection (Fig.4). All the patients were dignosed clinically as having congenital nasal dermoids and evaluated radiologically by CT scan of nose and paranasal sinuses (PNS) (Fig. 5). Nobody was found to have any intracranial extension of the sinus tract.

Operative Procedure: We selected a uniform protocol

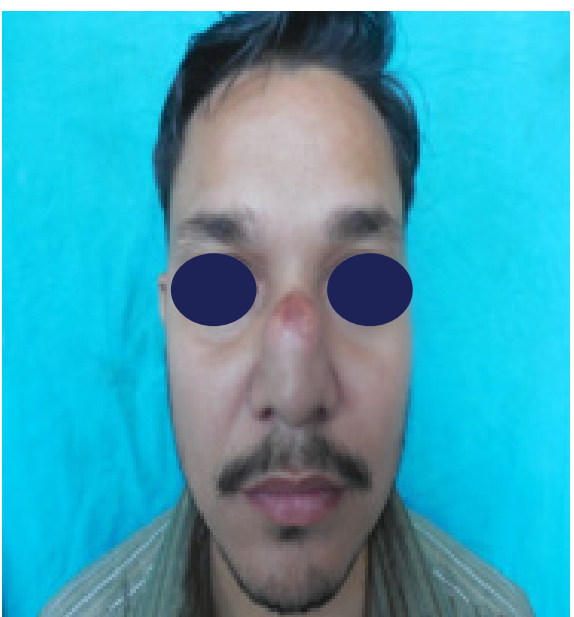

Fig.1. Dermoid cyst of nose with superadded skin inflammation

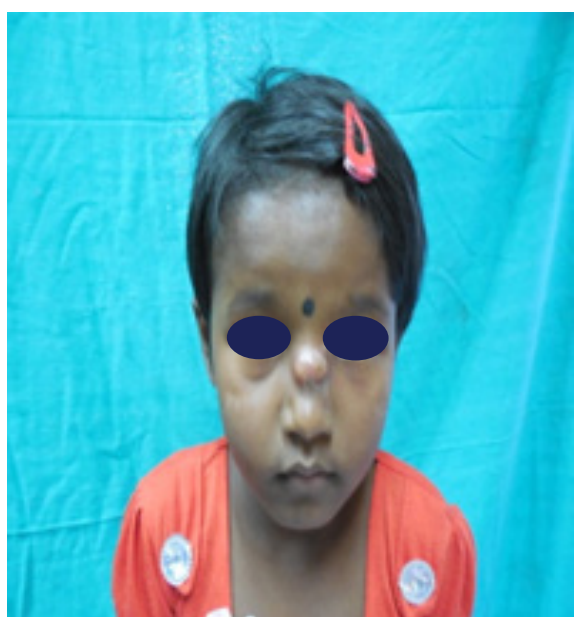

Fig.2. Globular dermoid cyst over dorsum of nose in a child

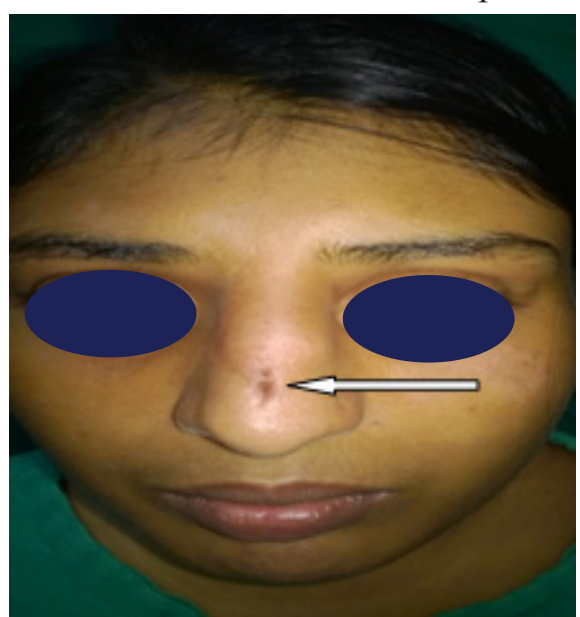

Fig.3. Midline nasal dermoid cyst with sinus(arrow) in a young female 


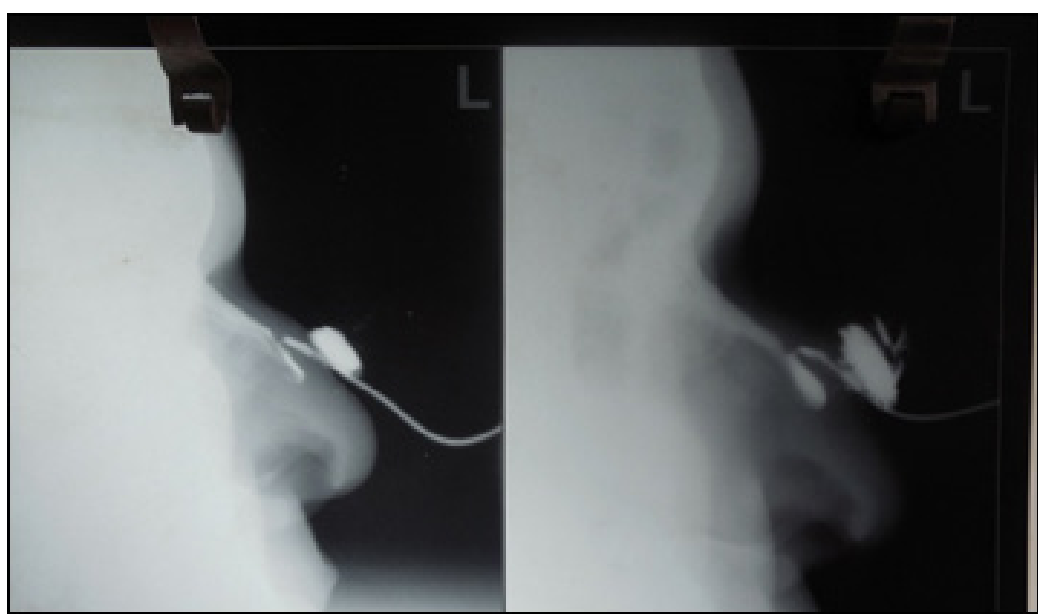

Fig.4. Sinogram to delineate the sinus tract in a dermoid sinus with cyst

for management of the cases with nasal dermoids. After treatment with antibiotics and incision-drainage wherever necessary, a CT scan of nose and PNS was done in every patient. After preparing the patients who were declared fit for GA, proper counselling and informed consent obtained and patients were taken up for definitive surgical excision of the dermoid cyst with sinus tract where exists.

Careful preoperative examination was done to plan excision. Probing of the sinus tract and injection of dye (methylene blue) just before incision helped to trace the tract during excision. Majority of the cases were dealt with an elliptical incision around the cyst or sinus tract (Fig. 6), two separate horizontal incisions were given along the Langer's line in cases where cyst and the tract coexisted. Incision was so fashioned that not to lose excess skin over the dorsum of nose, so that during repair adequate skin was present and finally the scar would not be under tension.

After giving incision, soft tissue dissection was carried out gently to separate the cyst or tract from surrounding tissue. In two cases we got multiple branches of the sinus tract, one ended in lacrimal sac and other ended at the root of nose while rest others with blind sacs. In cases of cysts, we incised the periosteum over nasal bones to dissect it en-bloc with the cyst. External rhinoplasty approach was not used in any of these patients nor any medial or lateral rhinotomy or attempt to fracture nasal

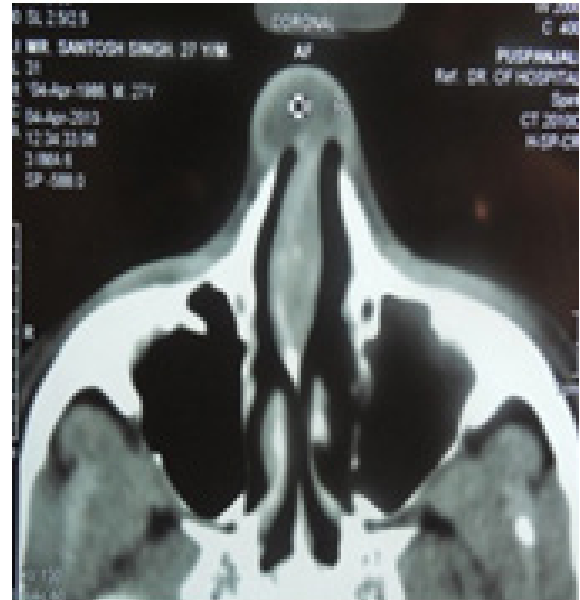

Fig.5. Axial CT scan of a dermoid showing splaying of nasal bones with a subcutaneous mass under nasal dorsum

bones to reunite them again were made as because all the patients except one were below 18 years of age which is generally believed to be the minimum age limit for the maturity of facial skeleton.

After total excision of the cyst or tract, soft tissue gaping were sutured by 4-0 synthetic absorbable (vicryl ${ }^{\circledR}$ ) suture and the skin was sutured by $4-0$ monofilament (ethilon ${ }^{\circledR}$ ) suture without tension.Zplasty closure was fashioned in one case to have best cosmetic result. Putting a drain is usually not needed

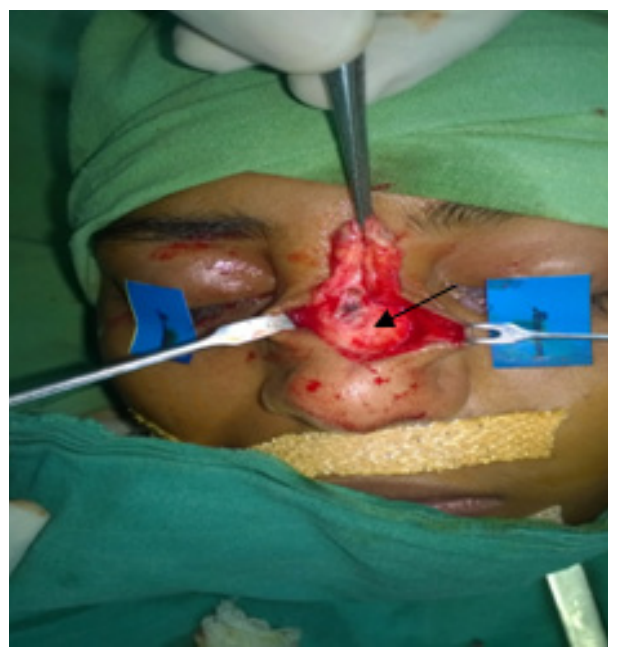

Fig.6. Excision of cyst becomes easy after delineation of sinus tract by injection of methylene blue dye 


\begin{tabular}{|c|c|c|c|c|c|c|c|c|c|c|c|c|c|}
\hline 8 & $\ddot{z}$ & $\stackrel{\circ}{\circ}$ & $\stackrel{̊}{ }$ & $\stackrel{̊}{\circ}$ & ค̊ & $\stackrel{̊}{\circ}$ & $\stackrel{̊}{\circ}$ & $\stackrel{̊}{\circ}$ & $\stackrel{ి}{ }$ & ค̊ & $\stackrel{\circ}{\circ}$ & $\stackrel{̊}{\circ}$ & $\stackrel{̊}{\circ}$ \\
\hline 高 & $\begin{array}{l}\Xi \\
\infty \\
=\end{array}$ & 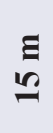 & $\stackrel{\Xi}{\Xi}$ & $\begin{array}{l}\Xi \\
\simeq\end{array}$ & $\underline{\Xi}$ & $\begin{array}{l}\Xi \\
\Xi\end{array}$ & $\underset{\beth}{\Xi}$ & $\underset{m}{\Xi}$ & 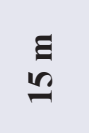 & $\begin{array}{l}\Xi \\
\beth\end{array}$ & $\underset{\Xi}{\Xi}$ & $\begin{array}{l}\Xi \\
\beth\end{array}$ & $\stackrel{\Xi}{\Xi}$ \\
\hline 䃕 & $\begin{array}{l}\grave{\bar{J}} \\
\tilde{D} \\
\frac{2}{30} \\
\overrightarrow{0}\end{array}$ & $\bar{z}$ & 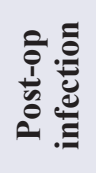 & $\overline{\bar{z}}$ & 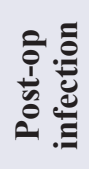 & $\overline{\bar{z}}$ & $\overline{\bar{z}}$ & $\overline{\bar{z}}$ & 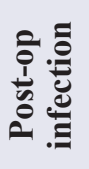 & 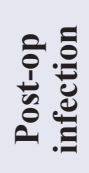 & $\overrightarrow{\mathbf{Z}}$ & $\bar{z}$ & 言 \\
\hline$\ddot{x}$ & + & + & + & + & + & + & + & + & + & + & + & + & + \\
\hline$\stackrel{\Xi}{Z}$ & & ' & 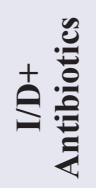 & 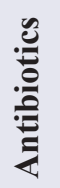 & 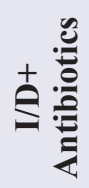 & ' & ' & ' & 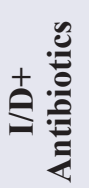 & ' & ' & ' & ' \\
\hline 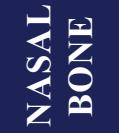 & 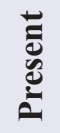 & 苛 & 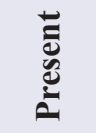 & 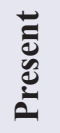 & 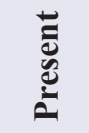 & 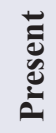 & 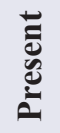 & 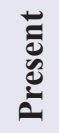 & 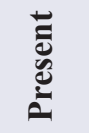 & 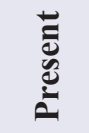 & 苛 & 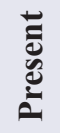 & 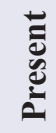 \\
\hline 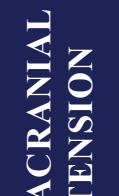 & $\bar{z}$ & $\overrightarrow{\mathbf{z}}$ & $\bar{z}$ & $\overline{\bar{z}}$ & $\bar{z}$ & $\overrightarrow{\mathbf{z}}$ & $\overline{\bar{z}}$ & $\bar{z}$ & $\bar{z}$ & $\bar{z}$ & $\vec{z}$ & $\overline{\bar{z}}$ & $\overline{\mathbf{z}}$ \\
\hline 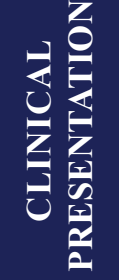 & 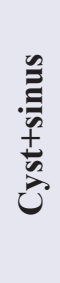 & 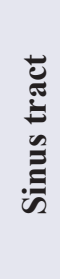 & 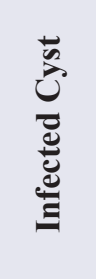 & 离 & 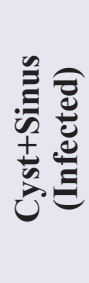 & 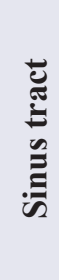 & 苑 & 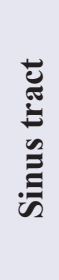 & 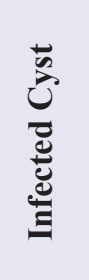 & 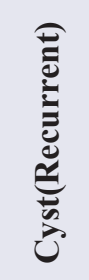 & $\tilde{\omega}$ & $\underbrace{ \pm}_{0}$ & 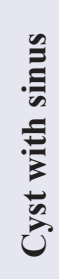 \\
\hline$\frac{\pi}{2}$ & $\sum_{\lambda}$ & $\sum_{F}^{\Sigma}$ & in & ल) & $\infty$ & $\sum_{i n}$ & $\stackrel{1}{0}$ & $\underset{r}{\sum}$ & $\Sigma_{6}$ & $\underset{N}{\Sigma}$ & $\sum_{b}$ & in & 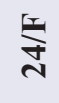 \\
\hline \# & - & $N$ & $m$ & + & n & 0 & $r$ & $\infty$ & $a$ & $\varrho$ & $=$ & $\simeq$ & 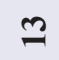 \\
\hline
\end{tabular}


if haemostasis is strictly maintained. Early removal of the sutures help to avoid ugly scar and the sutures were removed five days postoperatively. Suture removal was done under sedation specially in small children. Despite our honest effort one patient developed a bad scar postoperatively and an opinion from plastic surgeon was sought for scar revision which was planned as a second look surgery.

\section{Results}

In our study among 13 patients, seven were males and six were females. Seven patients presented with cysts, three patients presented with sinus and the rest three presented with both cyst and sinus. One patient presented to us with recurrence of the cyst, probably due to incomplete removal, two years after the previous surgery done elsewhere. Three patients required initial management with antibiotics and incision-drainage for infected nasal dermoid cysts which was followed by definitive surgery. No immediate complications such as major bleeding related to the surgery were observed. Minor complications such as postoperative infections were recorded in two lesions, and they were treated by antibiotics without any more sequelae. One patient developed a bad scar on nose postoperatively. All the results in this study were recorded in a proforma after at least one year follow up (Table I).

\section{Discussion}

As far as development of nose is concerned, it occurs through three distinct phases, (1) preskeletal phase- development of mesenchymal swelling surrounding external nasal placodes, (2) chondrocranial phase-development of cartilaginous framework and (3) ossification phase-influx of cellular elements and fusion of nasal skeletal elements. ${ }^{9}$ At about third week of fetal life, paired thickenings (the olfactory or nasal placodes) appear in the cranial ectoderm near the embryonic anterior neuropore. Invagination of these placodes results in formation of nasal pits, which as they deepen serve to delineate medial and lateral prominences of the frontonasal process.
Nasal dermoids are epithelial lined cavities or sinus tracts with variable number of skin appendages including hair follicles, sebaceous glands and eccrine glands that arise from clustures of epithelium trapped during ectodermal process. A true dermoid cyst may occur alone beneath the skin without a cutaneous opening or sinus superficial to nasal bones, appearing as slowly enlarging mass. The dermoid sinus with or without a cyst, on the other hand is an extensive lesion extending into nasal cartilage and bones. If skin remains attached to the fibrous tissues of the nasal capsule in the pre-nasal space or the ponticulus, a tract is formed between developing bones, many a times attached with dura, creating a tract between the nasal skin and the dura (Foramen caecum).

The sinus may also extend through foramen caecum between the frontal bones superiorly and the nasal bones inferiorly (the so-called ponticulus nasofrontalis). Post et al described an unusual presentation of extension of a NDSC into the frontal sinus itself requiring osteoplastic flap approach with a local midline incision. ${ }^{10}$ Nasal dermoids account for $3.7-12 \%$ of all dermoids of the head and neck and $1.1 \%$ of all the body dermoids. ${ }^{11}$ These are typically midline lesions, manifesting anywhere from the columellar base to the glabella. Clinical presentation may be single or multiple lesions in the form of a nasal pit, mass or a fusiform tract, often infected (presenting as an abscess) with or without hair and sebaceous materials. These are slightly more prevalent in males than in females and are usually visible at birth. In one study, four out of twenty-five (16\%) patients had intracranial extensions and 4\% patients presented with recurrence within three years of surgical excision. ${ }^{11}$ Re et al reported two cases of NDSCs with intracranial extensions which they managed with endoscopic endonasal procedures and, in their opinion, frequency of intracranial extensions of such cysts varies from $5 \%-45 \% .{ }^{3}$ In our series we could not get any such extensions.

Dermoids may extend intracranially and should be differentiated from encephaloceles both clinically and radiologically. A CT or magnetic resonance (MR) imaging aids in diagnosis as well as helps to determine the extent of intracranial involvement, if any. Radiological findings include fusiform swelling 
within the nasal septum, widening of the nasal vault and foramen caecum, bifid septum and crista galli, glabellar destruction, bony proliferation above cyst level, large ethmoidal cystic spaces etc.

Complications include abscess formation following infection (commonest), orbital/ periorbital cellulitis, osteomyelitis, meningitis and rarely brain abscess. There may also be sequlae of mass effect like new onset seizures. ${ }^{9}$

Craniofacial approach is required if intracranial extention is present. An incision and drainage with antibiotic treatment sometimes needed if secondary infection with abscess formation occurs, followed by excision of the tract. Various skin incisions like elliptical, $\mathrm{Y}, \mathrm{H}$ type with or without medial nasal osteotomies may be needed depending upon the case variety.

According to a study, very large lesions those in adults in whom underlying bone and cartilage have been damaged by prior surgery or erosion and in patients with known intracranial extension, a longitudinal zig-zag rhinotomy is used for wide exposure. ${ }^{11}$ The incisions are designed with limbs extending superiorly at angles greater than forty degrees but less than ninety degrees. Any fistulous opening is excised by fusiform excision. Scar prognosis is better than with a straight incision because the zig-zag are at less than ninety degrees to the relaxed skin tension lines running horizontally across the nose.

According to several studies ${ }^{1,5,9}$ open rhinoplasty approach with a stair-step columellar incision is one of the routes in many cases for the following advantages: ease of exposure, wide exposure of the nasal dorsum, controlled external osteotomies, ease of dorsal reconstruction, and wide exposure of the upper lateral cartilages and septum.

Timing of the surgery is decided considering the risk of infection or complications. Growth inhibition within the anterior facial skeleton can occur from extensive dissection, resulting in trauma to growth centres. Recurrence is attributed to incomplete excision. We got a single case of such recurrent dermoid cyst.

\section{Conclusion}

Although nasal dermoid sinus cysts are uncommon and complex lesions, they can be managed successfully with careful clinical assessment preoperative CT scans or MRI and appropriate surgery.

A minority of these patients will have intracranial extension, and the importance of preoperative CT scan is highlighted in our study to confirm the anatomy prior to surgery. Tailored definitive surgery including Z-plasty technique during skin closure and addressing defined anatomy and pathology, allows successful treatment with a low recurrence rate and fewer complications.

\section{References}

1. Morgan DW, Evans JN, Developmental nasal anomalies. J Laryngol Otol.1990; 104(5): 394-403

2. Chiu HH, Hsu WC, Shih JC, Tsao PN, Hsieh WS, Chou HC. The EXIT (ex utero intrapartum treatment) Procedure. J Formos Med. Assoc. 2008; 107(9):745-8

3. Re M, Tarclini P, Macri G, Pasquini E. Endonasal endoscopic approach for intracranial nasal dermoid sinus cyst in children; Int J Pediatr Otorhinolaryngol. 2012, 76(8):1217-22

4. Kennard CD, Rasmussen JE, Congenital nasal masses; diagnosis and management. J Dermatol Surg Oncol.1990, 16(11):1025-36

5. Wardinsky TD, Pagon RA, Kropp RJ, et al. Nasal dermoid sinus cysts; association with intracranial extention and multiple malformations. Cleft Palate Craniofac. J. 1991; 28(1):87-95

6. Hallak A, Jamjoom H, Hosseinzadeh T, Supernumerary nostrils; a case report \& review. Asthetic Plast Surg. 2001; 25(3):241-3

7. Broekmann ML, Hoving EW, Kho KH, et al. Nasal encephalocele in a child with Beckwith-Widemann Syndrome. J Neurosurg Pediatrics, 2008; 1(6):485-7

8. Loosee JE, Kirschner RE, Whitaker LA et al. Congenital nasa anomalies: a classification scheme. Plast. Reconstr. Surg. 2004; 113(2):676-89

9. Adil E, Huntley C, Choudhary A, Carr M. Congenital Nasal Obstruction: clinical and radiologic review. Eur J Pediatr, 2012; 17(4):641-50

10. Post G, McMains KC, Kountakis SE. Adult nasal dermoid sinus cyst. Am J Otolaryngol. 2005; 26(6):403-5

11. Blake WE, Chow CW, Holmes AD, Meara JG. Nasal dermoid sinus cysts-A retrospective review and discussion of investigation and management. Annals of Plast.surgery 2006; 57(5):535-40 